1970

\title{
Crystalline ionic solutions
}

\author{
Francis K. Fong \\ Robert L. Ford \\ Richard H. Heist \\ Fairfield University, rheist@fairfield.edu
}

Follow this and additional works at: https://digitalcommons.fairfield.edu/engineering-facultypubs (C1970 American Physical Society

The final publisher PDF has been archived here with permission from the copyright holder. https://doi.org/10.1103/PhysRevB.2.4202

\section{Repository Citation}

Fong, Francis K.; Ford, Robert L.; and Heist, Richard H., "Crystalline ionic solutions" (1970). Engineering Faculty Publications. 151.

https://digitalcommons.fairfield.edu/engineering-facultypubs/151

\section{Published Citation}

Fong, F. K., Ford, R. L., \& Heist, R. H. (1970). Crystalline ionic solutions. Physical Review B, 2(10), 4202. doi:10.1103/ PhysRevB.2.4202.

This item has been accepted for inclusion in DigitalCommons@Fairfield by an authorized administrator of DigitalCommons@Fairfield. It is brought to you by DigitalCommons@Fairfield with permission from the rightsholder(s) and is protected by copyright and/or related rights. You are free to use this item in any way that is permitted by the copyright and related rights legislation that applies to your use. For other uses, you need to obtain permission from the rights-holder(s) directly, unless additional rights are indicated by a Creative Commons license in the record and/or on the work itself. For more information, please contact digitalcommons@fairfield.edu. 


\title{
Crystalline Ionic Solutions*
}

\author{
Francis K. Fong, Robert L. Ford, and Richard H. Heist \\ Department of Chemistry, Purdue University, Lafayette, Indiana 47907
}

(Received 6 March 1970)

\begin{abstract}
Systems of crystalline ionic solutions containing impurity cations and associated compensation defects are described. Interactions between aliovalent ions and compensation defects give rise to configuration partition functions which predict a distribution in ion-defect pair separation distances at low temperatures. At elevated temperatures such ion-defect pairs dissociate, and the concept of pair formation more appropriately gives way to the concept of pair correlation functions. The relative sizes of the aliovalent ion and the host ion which it replaces are seen to exert a pronounced effect on pair distribution. Salient features of the theory are applied to the $\mathrm{KCl}: \mathrm{Sr}^{2+}, \mathrm{NaCl}: \mathrm{Mn}^{2+}, \mathrm{NaCl}: \mathrm{Sr}^{2+}, \mathrm{LiCl}: \mathrm{Mn}^{2+}$ and (alkaline-earth halide) : $\left(\right.$ rare earth) ${ }^{3+}$ systems. While the high-temperature treatment lends itself to comparisons with the DebyeHïckel theory for electrolytes, the low-temperature pair distribution theory is shown to be valid chiefly because of the specific nature of the crystalline ionic solutions.
\end{abstract}

\section{INTRODUCTION}

The law of equilibrium distribution which governs the low-temperature pair formation of divalent cations and cation vacancies in alkali halides ${ }^{1}$ and that of trivalent cations and interstitial anions in alkaline-earth halides ${ }^{2}$ has been useful in the interpretation and prediction of spectroscopic data on compensated lattices, ${ }^{2,3-6}$ in which aliovalent cations and lattice defects interact as ions of opposite unit charges in a crystalline solution. In general, $N_{i}$ unipositive ions and $N_{i}$ uninegative ions give rise to a total interaction energy, for a given configuration,

$$
\begin{aligned}
& U\left(\hat{R}_{1}, \hat{R}_{2}, \ldots, \hat{R}_{2 N_{i}}\right)=\sum_{1 \leq i<j \leq N_{i}}{ }^{*} u\left(\hat{R}_{i j}\right)
\end{aligned}
$$

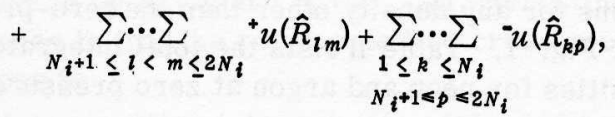

where $\hat{R}_{1}, \hat{R}_{2}, \ldots, \hat{R}_{2 N_{i}}$ are the position vectors of the $N_{i}$ cations and $N_{i}$ anions and ${ }^{+} u$ and $u$ denote repulsive and attractive interaction energies, respectively. At low temperatures at which only the attractive terms are important, it has been shown that the canonical configuration partition function

$$
Z=\sum_{\text {a.c. }} \exp \left[-U\left(\hat{R}_{1}, \hat{R}_{2}, \ldots, \hat{R}_{2 N_{i}}\right) / k T\right],
$$

where a.c. denotes all configurations, reduces to a product of "molecular" pair parition functions, such that ${ }^{1}$

$$
Z \cong \Omega_{p}\left(\sum_{l=1}^{l=l^{\circ}} g_{l} e^{-u\left(R_{l}\right) / k T}\right)^{N_{i}}=\Omega_{p} q_{p}^{N_{i}},
$$

where $g_{l}$ is the number of equivalent positions at which a defect (i.e., a uninegative ion) can be situated about an aliovalent cation (i.e., a unipositive ion) at a separation distance of $R_{l}$. The index $l$ denotes the number of nearest-neighbor separation, and $R_{l}=(2 l)^{1 / 2} a$ in alkali halides ${ }^{1}$ and $R_{l}$ $=(2 l-1)^{1 / 2} a$ in alkaline-earth halides, ${ }^{2} a$ being a characteristic lattice parameter for the host crystal. $^{1,2}$ The sum over $l$ is carried from $l=1$ to $l=l^{\prime}$, where $l^{\prime}$ is arbitrarily chosen such that $q_{p}$ does not change appreciably in summing beyond $l^{\prime}$. $\Omega_{p}$ is the number of ways of placing $N_{i}$ iondefect pairs in a finite host lattice. At a given temperature for which Eq. (3) is valid, the number $n_{l}^{*}$ of pairs of separation $R_{l}$ is

$$
n_{l}^{*}=N_{i} q_{p}^{-1} g_{l} e^{-u\left(R_{l}\right) / k T},
$$

which has been shown to be the case in the $\mathrm{KCl}$ : $\mathrm{Sm}^{2+1,3}$ and the $\mathrm{CaF}_{2}: \mathrm{RE}^{3+}$ ( $\mathrm{RE}$ denotes rare earth ${ }^{2}$ ) systems at appropriately low temperatures. At high temperatures at which the ion-defect pairs dissociate to an appreciable extent, Eqs. (3) and (4) become meaningless as we must include the repulsive terms in Eq. (1) in our evaluation of $Z$.

The purpose of the present work is threefold: (a) to generalize the treatment of ionic solutions in crystalline lattices by extending the consideration of $Z$ to high temperatures; (b) to extend the distribution calculation to several crystalline ionic solution systems and thereby examine the effect of the relative sizes of the aliovalent ion and the host ion upon the distribution of pair formation; and (c) to clarify the validity of Eq. (3), which results from a conceptual argument rather than a rigorous derivation. Differences as well as similarities between our treatment of the crystalline ionic solutions and that (e.g., the Debye-Hückel theory) of ionic solutions in fluid states will be emphasized. 


\section{HIGH-TEMPERATURE PARTITION FUNCTION AND PAIR CORRELATION FUNCTION}

In rewriting Eq. (2) for the configuration partition function at high temperature, and in taking into account all the attractive and repulsive terms, we define the following quantities as a measure of departure from ideality (1.e., the absence of interactions between the ions):

$$
\begin{aligned}
& { }^{+} f_{i j}=e^{++u\left(\hat{R}_{i j}\right) y_{k T}}-1, \\
& { }^{+} f_{l m}=e^{-+u\left(\hat{R}_{l m}\right) / k T}-1,
\end{aligned}
$$

and

$$
f_{k p}=e^{-u\left(\hat{R}_{k p}\right) / k T}-1,
$$

such that

$$
Z=\sum_{a_{0} c .} \Pi\left(1+{ }^{+} f_{i j}\right)\left(1+{ }^{+} f_{l m}\right)\left(1+{ }^{-} f_{k p}\right) .
$$

For a given configuration,

$$
\begin{aligned}
& e^{-U\left(\hat{R}_{1}, \hat{R}_{2}, \cdots, \hat{R}_{2 N_{i}}\right) / k T}=\prod\left(1+{ }^{+} f_{i j}\right)\left(1+{ }^{+} f_{l m}\right)\left(1+{ }^{-} f_{k p}\right) \\
& =1+\sum{ }^{+} f_{i j}+\sum{ }^{+} f_{l m}+\sum-f_{k p}+\sum{ }^{+} f_{i j}{ }^{+} f_{i^{\circ} j^{\circ}}+\sum{ }^{+} f_{l m}{ }^{+} f_{l^{\prime} m^{\circ}} \\
& +\sum{ }^{-} f_{k p}-f_{k^{\prime} p^{\circ}}+\sum{ }^{+} f_{i j}{ }^{-} f_{k p}+\sum{ }^{+} f_{l m} f_{k p}+\sum{ }^{+} f_{i j}^{+} f_{l m}
\end{aligned}
$$$$
\text { +higher terms , }
$$

where there are $\frac{1}{2} N_{i}\left(N_{i}-1\right)$ identical terms in $\Sigma^{+} f_{i j}$ which is identical to $\sum^{+} f_{l m}, N_{i}^{2}$ in $\sum f_{k p}, \frac{1}{8} N_{i}^{4}$ in $\sum^{+} f_{i j}{ }^{+} f_{i^{\circ}, j^{\circ}}$ which is identical to $\sum^{+} f_{l m}{ }^{+} f_{l^{\circ} m^{\circ}}, \frac{1}{2} N_{i}^{4}$ in $\sum f_{k p}^{-} f_{k^{\prime} p^{\circ}} \frac{1}{2} N_{i}^{4}$ in $\sum^{+} f_{i j}^{-} f_{k p}$ which is identical to $\sum^{+} f_{l m}-f_{k p}$, and $\frac{1}{4} N_{i}^{4}$ in $\sum^{+} f_{i j}{ }^{+} f_{l m}$. The meaning of the sum of ${ }^{+} f_{i j}$ over all configurations

$$
\sum_{\text {a.c. }}{ }^{+} f_{i j}=\sum_{\text {a.c. }}\left(e^{*^{+} u\left(\hat{R}_{i j}\right) / k T}-1\right)
$$

can be examined in the following manner: For a given fixed $\hat{R}_{i j}$, there are $\Omega^{\prime}$ ways of arriving at all possible ways of arranging the remaining $2\left(N_{i}-1\right)$ ions, such that

$$
\sum_{\text {a. c. }}^{*} f_{i j}=\Omega^{\prime} \sum_{\hat{R}_{i j}}\left(e^{*^{*} u\left(\hat{R}_{i j}\right) / k T}-1\right),
$$

where the sum is carried over all possible values for $\hat{R}_{i j}$. Fixing the position of the $i$ th ion as the center of our new coordinate system, we have $R_{i j}$ $=R_{j}$, the distance of separation of the $j$ th ion measured from the origin,

$$
\sum_{\text {a.c. }}^{+} f_{i j}=\Omega \sum_{R_{j}} g_{j}\left(e^{-+} u\left(R_{j}\right) / k T-1\right),
$$

where $g_{j}$ is the number of equivalent positions about the $i$ th ion (i.e., the origin) at $R_{j}$. The prime on $\Omega$ has been dropped due to the coordinate transformation. The determination of $\Omega$ can be made by realizing the fact that

$$
\Omega \sum_{j} g_{j}=\Omega_{0}\left(2 N_{i}, N\right),
$$

where $\Omega_{0}\left(2 N_{i}, N\right)$ is, for example, the total number of configurations for the random mixing of $2 N_{i}$ ions on $N$ available lattice sites as in $\mathrm{KCl}: \mathrm{Sm}^{2+}$

$$
\Omega_{0}\left(2 N_{i}, N\right)=N ! /\left(N_{i} !\right)^{2}\left(N-2 N_{i}\right) ! .
$$

Since $\sum_{j} g_{j}=N-1$, we obtain

$$
\Omega=N ! /\left(N_{i} !\right)^{2}\left(N-2 N_{i}\right) !(N-1) .
$$

Further analysis of the terms in Eq. (6) in terms of $\Omega, g_{j}, N_{i}, N,{ }^{+} u$, and $~ u$, readily leads to the following expression for the configuration partition function in the case $N_{i} / N \ll 1$ :

$$
\begin{aligned}
Z= & \Omega_{0}\left(2 N_{i}, N\right)\left(1+\frac{N_{i}}{(N-1)}\left[\sum_{j} g_{j}\left(e^{-+u\left(R_{j}\right) / k T}-1\right)\right.\right. \\
& \left.\left.+\sum_{p} g_{p}\left(e^{--u\left(R_{p}\right) / k T}-1\right)\right]\right)^{N_{i}}+\text { "other terms" , }
\end{aligned}
$$

where the contribution of the "other terms" becomes negligibly small ${ }^{7}$ for a dilute system in which which $N_{i} / N \ll 1$. Neglecting the "other terms" in Eq. (14), we write for the internal energy due to configuration interaction,

$$
\bar{U}=k T^{2} \frac{\partial \ln Z}{\partial T}=\frac{N_{i}^{2}}{z N} \sum_{l} g_{l}\left(e^{-u_{l} / k T}-e^{u_{l} / k T}\right)\left(u_{l}-T \frac{\partial u_{l}}{\partial T}\right),
$$

where

$$
z=1+\frac{N_{i}}{N}\left\{2 \sum_{l} g_{l}\left[\cosh \left(\frac{u_{l}}{k T}\right)-1\right]\right\}=1+\frac{N_{i}}{N} S
$$

and

$$
u_{l}={ }^{+} u\left(R_{l}\right)=-{ }^{-} u\left(R_{l}\right) .
$$

From Eq. (2), we obtain

$$
\begin{aligned}
\bar{U} & =\frac{k T^{2}}{Z} \frac{\partial}{\partial T} \sum_{\text {asco }_{0}} e^{-U\left(\hat{R}_{1}, \hat{R}_{2} \cdots, \hat{R}_{2 N_{i}}\right) / k T} \\
& =\frac{1}{Z} \sum\left(U-T \frac{\partial U}{\partial T}\right) e^{-U / k T} .
\end{aligned}
$$

Upon substitution of Eq. (1) in Eq. (17),

$$
\begin{aligned}
& \bar{U}=\frac{1}{Z} \sum_{\text {a o c. }}\left[\left[2 \sum_{i} \sum_{j}+u\left(\hat{R}_{i j}\right) e^{-U / k T}+\sum_{k} \sum_{p}-u\left(\hat{R}_{k p}\right) e^{-U / k T}\right]\right. \\
& \left.-T\left(2 \sum_{i} \sum_{j} \frac{\partial^{+} u\left(\hat{R}_{i j}\right)}{\partial T} e^{-U / k T}+\sum_{k} \sum_{p} \frac{\partial^{-} u\left(\hat{R}_{k p}\right)}{\partial T} e^{-U / k T}\right)\right] .
\end{aligned}
$$

Since there are $N_{i}\left(N_{i}-1\right) \sim N_{i}^{2}$ identical terms in $\sum_{i} \sum_{j}{ }^{+} u\left(\hat{R}_{i j}\right) e^{-U / k T}$ and $N_{i}^{2}$ identical terms in $\sum_{k} \sum_{p} u\left(\hat{R}_{k p}\right) e^{-U / k T}$, we rewrite Eq. (18) in the equivalent form (replacing $\hat{R}_{i j}$ by $\hat{R}_{12}$ and $\hat{R}_{k p}$ by 


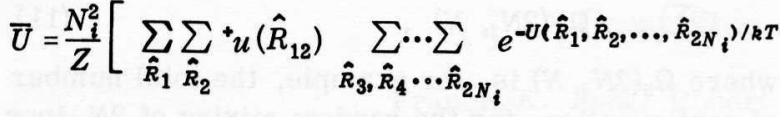

$$
\begin{aligned}
& +\sum_{\hat{R}_{1}} \sum_{\hat{R}_{\left(N_{i}+1\right)}}-u\left(\hat{R}_{1\left(N_{i}+1\right)}\right) \\
& \times \quad \sum \cdots \sum \quad e^{-U\left(\hat{R}_{1}, \hat{R}_{2}, \cdots, \hat{R}_{2 N_{i}}\right) / k T} \\
& \hat{R}_{2,} \hat{R}_{3}, \cdots, \hat{R}_{N_{i}} \\
& \hat{R}_{\left(N_{i}+2\right)}, \cdots, \hat{R}_{2 N_{i}} \\
& -T\left(\sum_{\hat{R}_{1}} \sum_{\hat{R}_{2}} \frac{\partial^{+} u\left(\hat{R}_{12}\right)}{\partial T} \sum_{\hat{R}_{3}, \hat{R}_{4}, \cdots, \sum_{2 N_{i}}} e^{-U\left(\hat{R}_{1}, \hat{R}_{2}, \cdots, \hat{R}_{2 N_{i}}\right) / k T}\right. \\
& +\sum_{\hat{R}_{1}} \sum_{\hat{R}_{\left(N_{i}+1\right)}} \frac{\partial^{-} u\left(\hat{R}_{1\left(N_{i}+1\right)}\right)}{\partial T} \\
& \left.\left.\times \sum_{\hat{R}_{2}, \hat{R}_{3}, \cdots, \hat{R}_{N i}} e^{-U\left(\hat{R}_{1}, \hat{k}_{2}, \cdots, \hat{R}_{2 N_{i}}\right) / k T}\right)\right] . \\
& \hat{R}_{\left(N_{i}+2\right)}, \cdots, \hat{R}_{2 N_{i}}
\end{aligned}
$$

We now define the generic distribution functions $\rho^{(2)}$ :

$$
\begin{aligned}
+\rho^{(2)}\left(\hat{R}_{12}\right) & =N_{i}^{2+} P^{(2)}\left(\hat{R}_{12}\right) \\
& =\frac{N_{i}^{2}}{Z} \sum_{\hat{R}_{3}, \hat{R}_{4}, \cdots \sum_{\hat{R}_{2 N_{i}}}} e^{-U\left(\hat{R}_{1}, \hat{R}_{2}, \cdots, \hat{R}_{2 N_{i}}\right) / k T},
\end{aligned}
$$

$$
\begin{aligned}
& -\rho^{(2)}\left(\hat{R}_{1\left(N_{i}+1\right)}\right)=N_{i}^{2}-P^{(2)}\left(\hat{R}_{1\left(N_{i}+1\right)}\right) \\
& =\frac{N_{i}^{2}}{Z} \sum_{\substack{\hat{R}_{2}, \hat{R}_{3}, \cdots R_{N_{i}} \\
\hat{R}_{\left(N_{i}+2\right)}, \cdots \hat{R}_{2 N_{i}}}} e^{-U\left(\hat{R}_{1}, \hat{R}_{2}, \cdots, \hat{R}_{2 N_{i}}\right) / k T},
\end{aligned}
$$

where ${ }^{+} P^{(2)}\left(\hat{R}_{12}\right)$ is the specific distribution function giving the probability of finding the second like ion at $\hat{R}_{2}$ with the first at $\hat{R}_{1}$, the sum being carried over all possible position vectors for the remaining $\left(2 N_{i}-2\right)$ ions, and ${ }^{\prime} P^{2)}\left(\hat{R}_{1\left(N_{i}+1\right)}\right)$ has the same meaning for two ions of opposite charges. The pair correlation function, or the radial distribution function is given as

$$
G\left(R_{12}\right)=\left(N / N_{i}\right)^{2} \rho^{(2)}\left(R_{12}\right) .
$$

Performing a coordinate transformation fixing a cation as the center of origin,

$$
{ }^{ \pm} G\left(R_{l}\right)=\left(N / N_{i}\right)^{2 \pm} \rho^{(2)}\left(R_{l}\right),
$$

where the + and - signs denote the charge of the second ion that is $R_{l}$ away from the origin. Upon substitution of Eq. (22) in Eq. (19), we obtain

$$
\bar{U}=\frac{N_{1}^{2}}{N} \sum_{l} u_{l}-T \frac{\partial u_{l}}{\partial T}\left[{ }^{+} G\left(R_{l}\right)-{ }^{-} G\left(R_{l}\right)\right]
$$

having made use of the identity

$$
\sum_{\hat{R}_{1}, \hat{R}_{2}} \equiv N \sum_{l} \text {. }
$$

Comparison of Eqs. (15) and (23) yields

$$
{ }^{ \pm} G\left(R_{l}\right)=z^{-1} g_{l} e^{\mp u_{l} \mid k T},
$$

which gives a measure of the probability of finding $\mathrm{a}+$ or - ion at a distance $R_{l}$ from the center of origin.

There is the probability $\left[N_{i} /(N-1)\right]^{ \pm} G\left(R_{\imath}\right)$ of placing one of the $N_{i}+$ or -ions at a distance $R_{l}$ from the central positive ion. Since there are $N_{i}$ positive ions, the total number ${ }^{ \pm} n_{l}^{*}$ of pair interactions at $R_{l}$ is given by

$$
{ }^{ \pm} n_{l}^{*}=\frac{N_{i}^{2}}{N-1}{ }^{*} G\left(R_{l}\right)=\frac{N_{i}^{2}}{(N-1) z} g_{l} e^{\mp u_{l} / k T} .
$$

The total number of attractive interactions at all values of $R_{l}$ is $N_{i}^{2}$, i. e. , from Eq. (25),

$$
\sum_{l}^{-} n_{l}^{*}=N_{i}^{2}=\frac{N_{i}^{2}}{(N-1) z} \sum_{l} g_{l} e^{-u} l / k T,
$$

such that

$$
\sum_{l} g_{l} e^{-u_{l} / k T}=(N-1) z=q .
$$

This is true only when $u_{l}<k T$ for most important values of $R_{l}$, when $q \sim(N-1)$ and $z \sim 1$. The resulting condition from the definition of $z$ in Eq. (16) is

$$
S=2 \sum_{l} g_{l}\left[\cosh \left(\frac{u_{l}}{k T}\right)-1\right] \ll \frac{N}{N_{i}} .
$$

In Sec. III, we shall make sample calculations of ${ }^{ \pm} n_{l}{ }^{*}$ according to Eq. (25).

\section{INTERACTION POTENTIAL AND CONVERGENCE}

In the evaluation of $Z$ in Eq. (14), we have ${ }^{*} u_{z}$ $= \pm e^{2} / \epsilon R_{l}$ (except for the nearest-neighbor interactions which are briefly described in Sec. IV), where $e$ is the electrostatic unit and $\epsilon$ is the macroscopic dielectric constant of the crystalline lattice. In neglecting the "other terms" in Eq. (14) in arriving at Eq. (25), however, we have in essence overlooked the presence of all the other ions in our consideration of the interactions between given pairs of ions. As a result of this, it can be easily shown that $S$ in Eq. (28) diverges unless a screen $e^{-b R_{l}}$ is invoked for Coulombic interactions at large $R_{l}$ as in the Debye-Hückel theory, where $b=\left(8 \pi N_{i} e^{2} / \epsilon V k T\right)^{1 / 2}, V$ being the volume of the system.

In the numerical evaluation of $Z$, we need to define $u_{l}$ for all values of $R_{l}$. We assume that when $u_{l} / k T \leq 0.1, u_{l}=\left(e^{2} / \epsilon R_{l}\right) e^{-b R_{l}}$. At $l=1$ and 2 , polarization effects as well as Coulombic in- 
teractions must be taken into consideration in the evaluation of $u_{1}$ and $u_{2}{ }^{1}, 8$ At $l=3$, we assume purely Coulombic interaction which is reasonable in view of the previous investigations. ${ }^{1,2}$ In the intermediate region $R_{3} \leq R_{l} \leq R_{m}$, where $R_{m}$ is the value of $R_{l}$ at which $u_{m} / k T \sim 0.1, u_{l}$ is assumed to have the following form

$$
u_{l}=\frac{e^{2}}{\epsilon R_{l}} \exp \left[-b R_{l} e^{-\left(R_{m}-R_{l}\right)\left(R_{l}-R_{3}\right)-1}\right],
$$

which provides a smooth interpolation from the screened Coulombic interaction at $R_{\imath} \geq R_{m}$ to the pure Coulombic interaction at $R_{3}$. By employing the appropriate lattice descriptions for $g_{l}$ as a function of $l,{ }^{1,2}$ the configuration partition function $Z$ in Eq. (14) has been evaluated in sum up to $l=1350\left(R_{1350}=163.16 \AA\right)$ with a CDC 6500 computer for the $\mathrm{CaF}_{2}: \mathrm{RE}^{3+}\left(\epsilon=6.7, u_{1}=-0.477 \mathrm{eV}\right.$, and $\left.u_{2}=-0.300 \mathrm{eV}\right)^{2}$ and the KCl:Sr${ }^{2+}\left(\epsilon=5.03, u_{1}=\right.$ $-0.39 \mathrm{eV}$, and $\left.u_{2}=-0.50 \mathrm{eV}\right)^{9}$ systems. The dependence of

$$
z=\left[\Omega_{0}^{-1}\left(2 N_{i}, N\right) Z\right]^{1 / N_{i}}
$$

on $N_{i}$ and $T$ is given in Fig. 1 in the region for $1 \leq z \leq 1$.2. Some typical calculations are listed in Table I. From Eq. (25), ${ }^{ \pm} n_{l}^{*}$ has been calculated for $\mathrm{CaF}_{2}: \mathrm{RE}^{3+}$ at $T=900,1100,1300,1500$, and $1700 \mathrm{~K}$ with $N_{i}=10^{17} \mathrm{~cm}^{-3}$. The results of this calculation are shown in Figs. 2 and 3 for $l \leq 21$. In sharp contrast with the low-temperature distri-

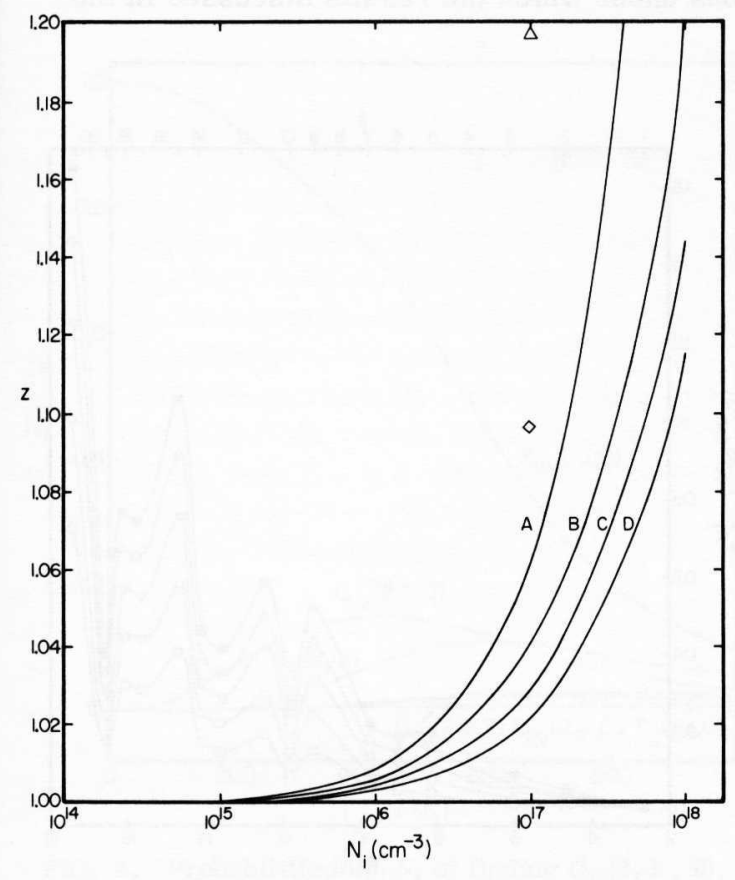

FIG. 1. Variation of $z$ with $N_{i}$ at $1100 \mathrm{~K}$ (A), $1300 \mathrm{~K}$ (B), $1500 \mathrm{~K}(\mathrm{C})$, and $1700 \mathrm{~K}$ (D) for $\mathrm{CaF}_{2}: \mathrm{RE}^{3+}$. The symbols $\diamond$ and $\triangle$ represent calculations for the $\mathrm{CaF}_{2}: \mathrm{RE}^{3+}$ and $\mathrm{KCl}: \mathrm{Sr}^{2+}$ systems at $900 \mathrm{~K}$, respectively.
TABLE I. Some sample calculations of $R_{m}, S=2 \sum_{l} g_{l}$ [cosh $\left.\left(\mu_{l} / k T\right)-1\right]$, and $z=1+\left(N_{i} / N\right) S$ for $\mathrm{KCl}: \mathrm{Sr}^{2+}$ and

\begin{tabular}{|c|c|c|c|c|c|}
\hline System & $\begin{array}{c}T \\
(\mathrm{~K})\end{array}$ & $\begin{array}{c}N_{i} \\
\left(\mathrm{~cm}^{-3}\right)\end{array}$ & $\begin{array}{l}R_{m} \\
(\AA)\end{array}$ & $\begin{array}{c}S \\
\left(10^{3}\right) \\
\end{array}$ & $z$ \\
\hline $\mathrm{KCl}: \mathrm{Sr}^{2+}$ & 900 & $10^{17}$ & 118.17 & 35.613 & 1.198 \\
\hline \multirow[t]{8}{*}{$\mathrm{CaF}_{2}: \mathrm{RE}^{3+}$} & 900 & $10^{17}$ & 110.35 & 24.684 & 1.100 \\
\hline & 1100 & $10^{17}$ & 103.68 & 15.179 & 1.062 \\
\hline & 1300 & $10^{17}$ & 97.50 & 9.888 & 1.040 \\
\hline & 1500 & $10^{16}$ & 128.02 & 10.577 & 1.004 \\
\hline & 1500 & $10^{17}$ & 91.85 & 7.240 & 1.029 \\
\hline & 1700 & $10^{15}$ & 135.19 & 9.141 & 1.000 \\
\hline & 1700 & $10^{16}$ & 117.18 & 8.084 & 1.003 \\
\hline & 1700 & $10^{17}$ & 86.69 & 5.569 & 1.023 \\
\hline
\end{tabular}
$\mathrm{CaF}_{2}: \mathrm{RE}^{3+}$. The sum $S$ is carried to $l=1350$.

bution curves, ${ }^{2}$ in which practically all the cations are paired with associated compensation defect anions, we observe from Fig. 2 that in the hightemperature case under conditions specified above, only a small fraction $\left(10^{-3}-10^{-2}\right)$ of the cations are in close association with the compensation defects. ${ }^{+} n_{l}{ }^{*}$ is several orders of magnitude lower than - $n_{l}^{*}$ at small $l$, which is to be expected. Clearly, ${ }^{*} n_{l}^{*} /{ }^{*} n_{l}^{*}$ increases exponentially with $T$, approaching unity as $Z \rightarrow \Omega_{0}\left(2 N_{i}, N\right)$. Moreover, at $R_{l} \gg R_{m}$,

$$
-n_{l}^{*} \cong{ }^{*} n_{l}^{*} \cong\left[N_{i}^{2} /(N-1) z\right] g_{l},
$$

which follows from Eq. (25) since $u_{l} / k T \sim 0$ at $R_{l}$ $\gg R_{m}$. For $N_{i}=10^{17} \mathrm{~cm}^{-3},-n_{l}^{*} \sim{ }^{+} n_{l}^{*} \sim 10^{12} \mathrm{~g}_{l}$.

\section{LOW-TEMPERATURE PAIR DISTRIBUTION CURVES}

The results of the high-temperature treatment given in Secs. II and III differ in a fundamental manner from those of the low-temperature treatment. From the high-temperature treatment, the concept of pair correlation functions evolves which leads to the total number ${ }^{*} n_{l}^{*}$ of pair interactions at a distance $R_{l}$ between the interacting ions [Eq. (25) and Figs. 2 and 3]. The effect of the presence of other ions about a given cation in the high-temperature treatment thus, in essence, averages out isotropically, so that the cubic environment of the host lattice becomes the dominant perturbation for the crystal field symmetry. In the low-temperature limit, however, the picture is one of cation-defect pairs, and the cubicity about the cation is lowered to some symmetry prescribed by the position of the defect relative to the cation. In this section we shall first extend the low-temperature distribution calculation to several crystalline ionic solutions in order to observe certain trends. The fundamental differences between the high- and low-temperature treatments will be discussed in the closing paragraphs in Sec. V.

At low temperature and high dilution, screening due to other impurity-defect pairs may be ne- 


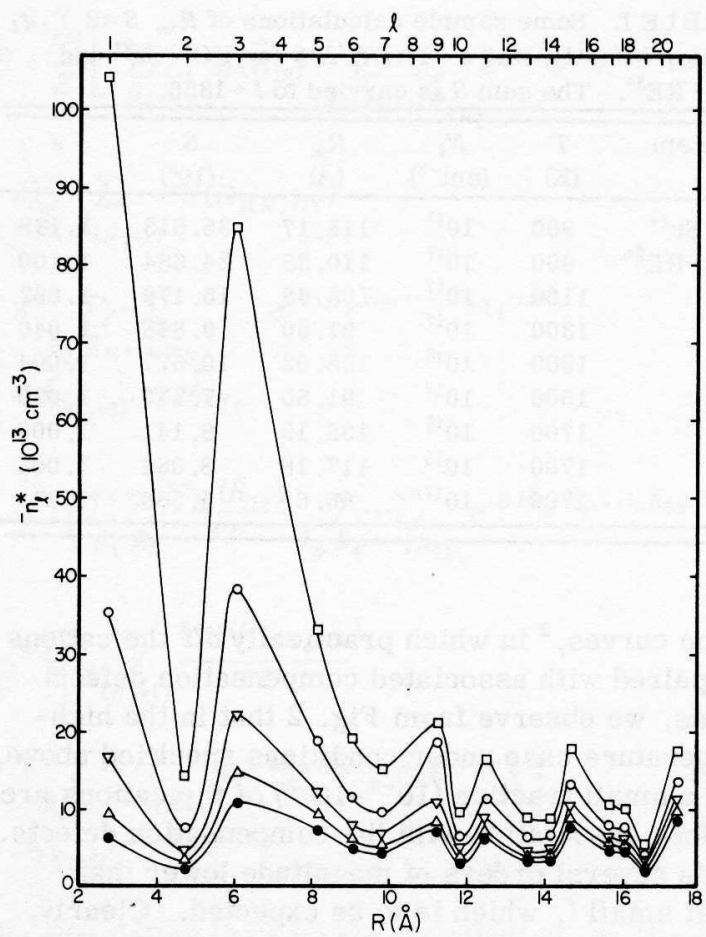

FIG. 2. Number ${ }^{-} n_{t}^{*}$ of attractive pair interactions for $l \leqslant 21$ at $900 \mathrm{~K}(\square), 1100 \mathrm{~K}(\mathrm{C}), 1300 \mathrm{~K}(\nabla), 1500 \mathrm{~K}(\diamond)$, and $1700 \mathrm{~K}(\bullet)$. The curves connecting the points are shown as a visual guide and are not to be taken as part of the calculations.

glected, ${ }^{1}$ and the interaction energy $u_{l}$ may be regarded as purely Coulombic for all but the first and second nearest-neighbor (nn) pairs. ${ }^{8}$ The departure from a purely Coulombic potential at small values of $l$ is due to the repulsive interaction from electron overlap and to the polarization of the lattice about the impurity-defect pair. Values for $u_{1}$ and $u_{2}$, the first and second $\mathrm{nn}$ interaction energies, have been calculated or measured for the following systems: $\mathrm{NaCl}: \mathrm{Sr}^{2+}\left(u_{1}=-0.45 \mathrm{eV}\right.$, $\left.u_{2}=-0.41 \mathrm{eV}\right),{ }^{8} \mathrm{NaCl}: \mathrm{Mn}^{2+}\left(u_{1}=-0.3 \mathrm{eV}, u_{2}=\right.$ $-0.35 \mathrm{eV}),{ }^{9}$ and LiCl: $\mathrm{Mn}^{2+}\left(u_{1}=-0.3 \mathrm{eV}, u_{2}=\right.$ $-0.26 \mathrm{eV}) .^{9}$ Using these values and Eq. (4), we have calculated site symmetry distributions with $q_{p}$ summed to $l^{\prime}=200$ in the manner previously described. ${ }^{1}$ The results are shown in Figs. 4-6.

From Figs. 4-6 and the distribution curves previously determined for the $\mathrm{KCl}: \mathrm{Sr}^{2 *}$ system, ${ }^{1}$ we observe that as the size of the impurity ion decreases, or conversely as the lattice spacing of the host increases, the second $n n$ compensation becomes increasingly more probable. Although the unavailability of $u_{1}$ and $u_{2}$ for (alkaline-earth halide): $\mathrm{RE}^{3+}$ systems other than $\mathrm{CaF}_{2}{ }^{2}$ deprives us of detailed calculations of site symmetry distributions, electron paramagnetic resonance (EPR) in- vestigations have revealed a similar trend in these systems. ${ }^{10,11}$ For example, first $n$ compensation was not observed for the $\mathrm{BaF}_{2}: \mathrm{Yb}^{3+10}$ and

$\mathrm{BaF}_{2}: \mathrm{Gd}^{3+11}$ systems, while the probability for the first $\mathrm{nn}$ compensation in $\mathrm{CaF}_{2}: \mathrm{RE}^{3+}$ systems is found to be predominant. ${ }^{2}$ An explanation of this trend can be given by considering one of the terms contributing to the binding energies of the first and second nn pairs. This term, introduced by Brauer, ${ }^{12}$ considers the effect of the size of the impurity ion on the binding energy of the pair. It accounts for the fact that the perturbing impurity ion is not only a source of electrostatic polarization, but, as its ionic radius may be quite different from that of the cation it has replaced, it may also be a source of displacement polarization. Each ion in the surrounding lattice undergoes a purely elastic displacement and consequently produces a displacement dipole which has the net effect of altering the binding energies of the impurity-defect pairs. When the impurity ion decreases in size, the $\mathrm{nn}$ shell of host anions, in particular, will be displaced inward. These displacement dipoles will have the effect of increasing the shielding between the impurity ion and its first $\mathrm{nn}$ defect compensations, thus reducing $u_{1}$. The quantity $u_{2}$, on the other hand, will increase as the second $\mathrm{nn}$ defect moves closer to the impurity ion due to the inward displacement of the $\mathrm{nn}$ host anions, thus accounting for the observed changes in the relative abundance of the first two nn pairs. The conditions under which the results discussed in the

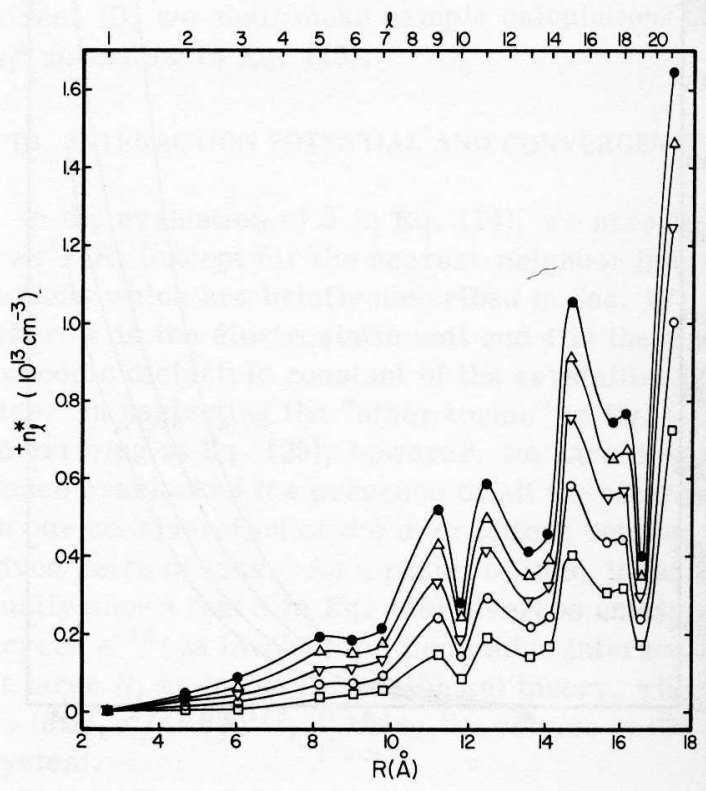

FIG. 3. The number ${ }^{+} n_{l}^{*}$ of repulsive pair interactions for $l \leqslant 21$ at $900 \mathrm{~K}(\square), 1100 \mathrm{~K}(0), 1300 \mathrm{~K}(\nabla), 1500 \mathrm{~K}$ $(\diamond)$, and $1700 \mathrm{~K}(\bullet)$. 
present section are valid will be elaborated in Sec. V.

\section{v. DISCUSSION}

The high-temperature treatment given in this work is valid under the condition Eq. (28), which will be satisfied at high temperature and high dilution. In the Debye-Hückel theory, the basic assumption is that ${ }^{ \pm} G(R)=e^{\mp u(R) / k T}$ which is, in essence, the same statement as Eq. (24). Our hightemperature treatment thus lends itself to comparison with the Debye theory, except that the discreteness of the crystalline lattice provides us with a unique handle on the evaluation of the pair correlation functions ${ }^{ \pm} G\left(R_{l}\right)$ and ${ }^{*} n_{l}^{*}$.

The low-temperature treatment ${ }^{1}$ reviewed in Sec. I and further elaborated in Sec. IV, on the other hand, is completely different in nature. If equilibrium conditions are attained at finite ionic concentrations, ions of opposite charges will pair as temperature decreases through attractive Coulombic interactions. At sufficiently low temperatures these ion pairs will cluster through higher polar interactions such that precipitation eventually results. This is the case, for example, in an aqueous solution of $\mathrm{KCl}$. In crystalline ionic solutions of the type described in the present paper, however, true equilibrium is not readily attainable at low temperatures due to the low mobilities of the aliovalent cations. These cations are, in fact, "frozen" in a metastable equilibrium characteristic

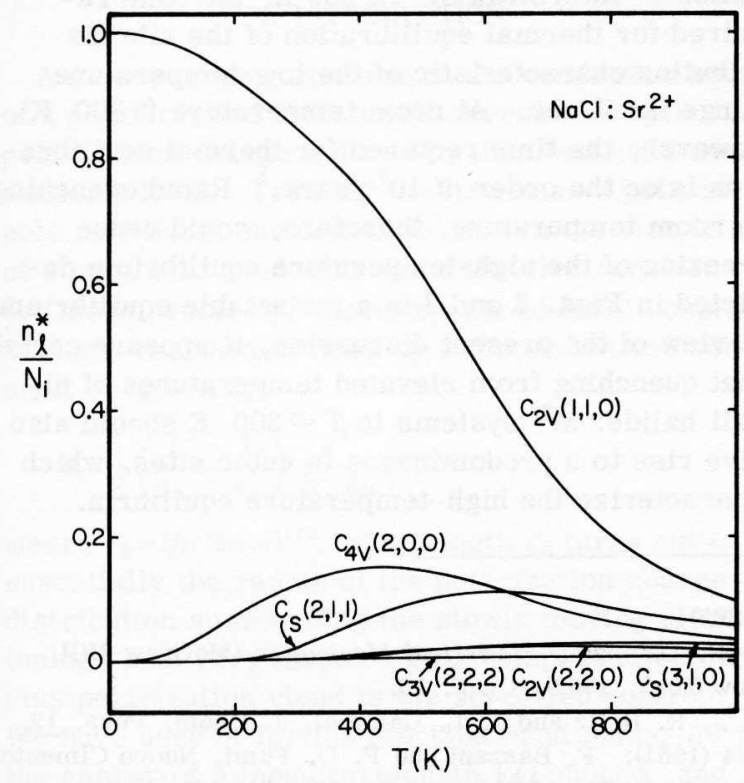

FIG. 4. Probabilities $n_{l}^{*} / N_{i}$ of finding $C_{2 v}(1,1,0)$, $C_{4 v}(2,0,0), C_{s}(2,1,1), C_{2 v}(2,2,0), C_{s}(3,1,0)$, and $3_{3 v}(2,2,2)$ sites in $\mathrm{NaCl}: \mathrm{Sr}^{2+}\left(\mathrm{Sr}^{2+}\right.$ ionic radius $=1.13 \AA$; $\mathrm{Na}^{+}$ionic radius $\left.=0.95 \AA\right)$. The symmetry notations have been explained in Ref. 1 .

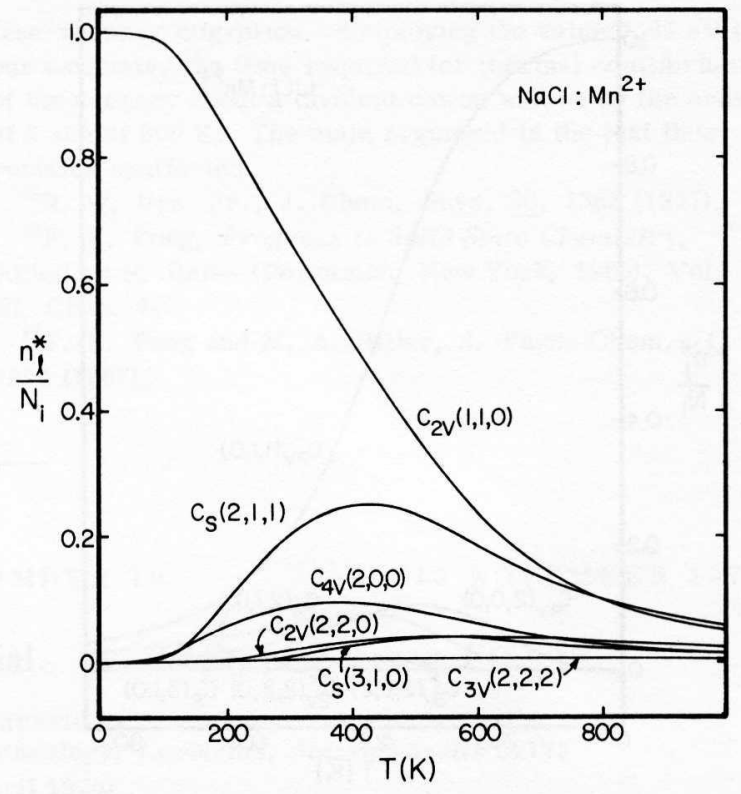

FIG. 5. Probabilities $n_{l}^{*} / N_{i}$ of finding $C_{2 v}(1,1,0)$, $C_{4 v}(2,0,0), C_{s}(2,1,1), C_{2 v}(2,2,0), C_{s}(3,1,0)$, and $C_{3 v}(2,2,2)$ sites in $\mathrm{NaCl}: \mathrm{Mn}^{2+}\left(\mathrm{Mn}^{2+}\right.$ ionic radius $=0.8 \AA$; $\mathrm{Na}^{+}$ionic radius $=0.95 \AA$ ).

of some higher temperature, in which they are well dispersed throughout the entire lattice. The associated defects (i.e., the anions), on the other hand, possess much greater mobilities so that they can equilibrate through attractive Coulombic forces in pair formation with the cations. For example, the $\mathrm{K}^{+}$vacancy in $\mathrm{KCl}$ has a migrational activation energy of $0.63 \mathrm{eV} .^{9}$ Assuming the value $10^{14}$ given $^{13}$ for the preexponential frequency factor for the jump of a $\mathrm{K}^{+}$vacancy from one lattice position to an adjacent one, the time required for thermal equilibration of the vacancy about a divalent cation will be on the order of $10^{-3} \mathrm{sec}$ at $300 \mathrm{~K}_{\circ}{ }^{14}$ We thus visualize the formation of cation-defect pairs which cannot aggregate through higher polar attractions at low temperatures. In some proper temperature range, therefore, we have $N_{i}$ pairs whose mutual interactions are negligibly small (at sufficiently high dilution), and Eq. (3) results. The conceptual model depicted in Eq. (3) requires that the ensemble of ions is divided into $N_{i}$ cells, each of which containing one ion-defect pair. The "molecular" pair partition function $q_{p}$ must be summed over all $\left(N / N_{i}-1\right)$ lattice points per cell. The model will be valid if the occupation probability is significant only for the first few nn positions, i.e.,

$$
q_{p}=\sum_{l=1}^{l=1} g_{l} e^{-u_{l} / k T} \gg \frac{N}{N_{i}} .
$$

For example, $q_{p} \sim 10^{8}$ and $10^{5}$ at 300 and $500 \mathrm{~K}$, respectively, for $\mathrm{KCl}: \mathrm{M}^{2+}$. For $N_{i}=10^{17} \mathrm{~cm}^{-3}, \mathrm{~N} /$ 


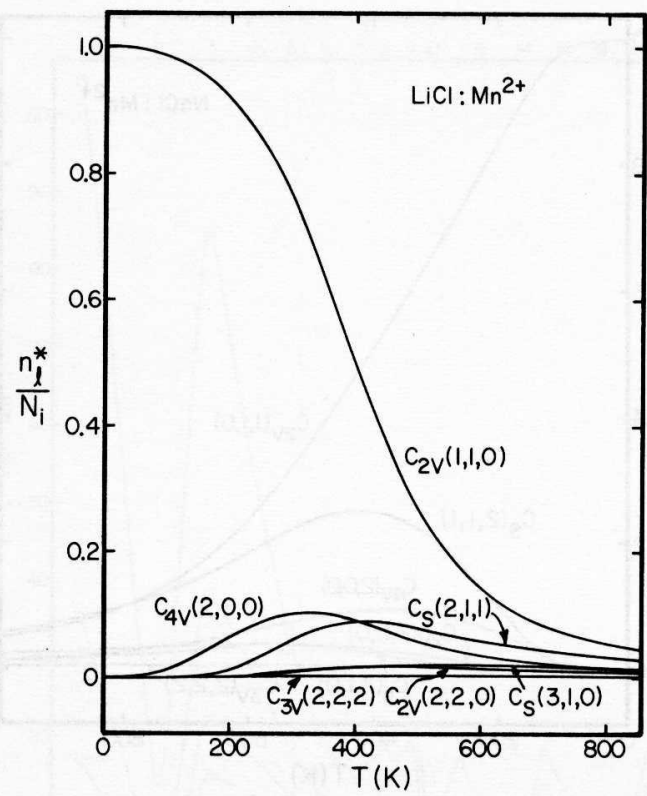

FIG. 6. Probabilities $n_{l}^{*} / N_{i}$ of finding $C_{2 v}(1,1,0)$, $C_{4 v}(2,0,0), C_{s}(2,1,1), C_{2 v}(2,2,0), C_{s}(3,1,0)$, and $C_{3 v}(2,2,2)$ sites in $\mathrm{LiCl}: \mathrm{Mn}^{2+}\left(\mathrm{Mn}^{2+}\right.$ ionic radius $=0.8 \AA$; $\mathrm{Li}$ ionic radius $=0.60 \AA$ ).

$N_{i} \sim 10^{3}$, and Eq. (3) is thus valid for $T \leqslant 500 \mathrm{~K}$ if pair formation equilibrium is attained. (At $T$ $\ll 300 \mathrm{~K}$ even the $\mathrm{K}^{+}$vacancy mobility will be too low for thermal equilibration.) At higher dilution, Eq. (3) will be valid at lower temperatures.

Finally, we return to the high-temperature results in an assessment of their applicability to real systems. The calculations have been made at sufficiently high dilution $\left(10^{17}-10^{18} \mathrm{~cm}^{-3}\right)$ and temperatures $(T \geq 900 \mathrm{~K})$ in order to ascertain the condition stated in Eq. (28). The concentrations of intrinsic defects were ignored in all our calculations for the sake of simplicity. In reality, however, these concentrations are by no means negligible at $T \geq 900 \mathrm{~K}$. In $\mathrm{KCl}$ and $\mathrm{CaF}_{2}$, for example, the intrinsic defect concentrations are on the order of $10^{16} \mathrm{~cm}^{-313}$ and $10^{17} \mathrm{~cm}^{-315},{ }^{16}$ at $900 \mathrm{~K}$, respectively. It is therefore necessary to extend our treatment to include the interactions due to the presence of intrinsic defects in the evaluation of
$Z$, which presents no serious difficulties. At $T$ $>900 \mathrm{~K}$, however, the intrinsic defect concentrations in $\mathrm{CaF}_{2}$ exceed the range in which Eq. (28) is valid, being as high as $\sim 10^{20}$ at $T=1300 \mathrm{~K}$. An adequate treatment of such a situation would require an inclusion of the "other terms" in Eq. (14). The results reported in Secs. II and III nevertheless represent a quantitative, albeit idealized, approach to the fairly complex problem of interacting dissociated ions in a periodic crystalline lattice. Equally important is the fact that through the contrast between these results and those obtained through the low-temperature partition function $Z$ of Eq. (3), several novel features of the pair distribution theory have been elucidated. The spectroscopic observation of the predominance of "cubic" sites in $\mathrm{CaF}_{2}: \mathrm{RE}^{3+}$ systems when they are quenched $^{2}$ from elevated temperatures is, in fact, experimental evidence of the results shown in Figs. 2 and 3. At $T=900 \mathrm{~K}$ and $N_{i}=10^{17} \mathrm{~cm}^{-3}$, only $\sim 2 \times 10^{15}-\mathrm{cm}^{-3}$ ion-defect pairs are within $l \leq 21$ (Fig. 2). Since the compensation crystal field potential varies as $R_{i}^{(k+1)}$ where $k>0$ is the rank of the spherical harmonic in the corresponding crystal field potential expansion, the crystal field effect of the more distant compensation sites would be negligibly small as the cubic environment of the $\mathrm{RE}^{3+}$ ion becomes the dominant factor in the spectroscopic observations. The predominance of cubic sites in $\mathrm{CaF}_{2}$ (which is not observed in the $\mathrm{KCl}: \mathrm{M}^{2+}$ systems ${ }^{1,3-6}$ ) arises from the high migrational activation energy $1.51 \mathrm{eV}^{17}$ of the compensation $\mathrm{F}^{-}$interstitials. At $600 \mathrm{~K}$, the time required for thermal equilibration of the site distribution characteristic of the low-temperature range is $\sim 1$ sec. At room temperature $(\sim 300 \mathrm{~K})$, however, the time required for thermal equilibration is on the order of $10^{7}$ years. ${ }^{2}$ Rapid quenching to room temperature, therefore, would cause freezing of the high-temperature equilibrium depicted in Figs. 2 and 3 in a metastable equilibrium. In view of the present discussion, it appears certain that quenching from elevated temperatures of alkali halide: $\mathrm{M}^{2 *}$ systems to $T \ll 300 \mathrm{~K}$ should also give rise to a predominance in cubic sites, which characterize the high-temperature equilibria.
*This research was supported under the institutional ARPA Grant No. SD102.

${ }^{1}$ F. K. Fong, Phys. Rev. 187, 1099 (1969).

${ }^{2}$ R. H. Heist and F. K. Fong, Phys. Rev. B 1,2970 (1970).

${ }^{3}$ F. K. Fong, Phys. Rev. B 1, 4157 (1970).

${ }^{4}$ F. K. Fong and J. C. Bellows, Phys. Rev. B 1,4240 (1970).

${ }^{5}$ F. K. Fong, R. H. Heist, C. R. Chilver, J. C. Bellows, and R. L. Ford, J. Luminescence 1, 889 (1969).

${ }^{6}$ F. K. Fong and E. Y. Wong, Phys. Rev. $\underline{162}, 348$
(1967).

${ }^{7}$ N. Davidson, Statistical Mechanics (McGraw-Hill, New York, 1962), Chap. 15.

${ }^{8}$ J. R. Reitz and J. L. Gammel, J. Chem. Phys. 19 894 (1951); F. Bassani and F. G. Fumi, Nuovo Cimento 11, 274 (1954); M. P. Tosi and G. Airoldi, ibid. $\underline{8}, 584$ (1958).

${ }^{9}$ G. D. Watkins, Phys. Rev. $\underline{113}, 79$ (1959); 113,91 (1959).

${ }^{10} \mathrm{U}$. Ranon and A. Yariv, Phys. Letters 9, 17 (1964).

${ }^{11} \mathrm{~J}$. Sierro, Phys. Letters $\underline{4}, 178$ (1963). 
${ }^{12}$ P. Brauer, Z. Naturforsch. $\underline{6 A}, 255$ (1951).

${ }^{13}$ R. W. Dreyfus and A. S. Norwick, Phys. Rev. 33 , 437 (1962).

${ }^{14}$ There is some question in this estimate due to the uncertainty in the migrational barrier for the $\mathrm{K}^{+}$vacancy migration. The value $0.63 \mathrm{eV}$ given by Watkins (Ref. 9) corresponds to the migration of a $\mathrm{K}^{+}$vacancy in the close proximity of the divalent cation. In an ionic conductivity experiment $\mathrm{H}$. W. Etzel and R. J. Maurer [J. Chem.

Phys. 18, $1003(1950)$ ] reported the value $0.85 \mathrm{eV}$ for the migrational barrier of $\mathrm{Na}^{+}$vacancy in $\mathrm{NaCl}$. The higher value reported by these authors probably corresponds to free-vacancy migration. Employing the value $0.85 \mathrm{eV}$ in our estimate, the time required for thermal equilibrium of the vacancy about a divalent cation will be on the order of $2 \mathrm{sec}$ at $300 \mathrm{~K}$. The main argument in the text thus remains unaffected.

${ }^{15}$ R. W. Ure, Jr., J. Chem. Phys. 26, 1363 (1957).

${ }^{16}$ F. K. Fong, Progress in Solid State Chemistry, edited by H. Reiss (Pergamon, New York, 1966), Vol. III, Chap. 4.

${ }^{17}$ F. K. Fong and M. A. Hiller, J. Phys. Chem. 71, 2854 (1967). 